\title{
HISTÓRIA DA EDUCAÇÃO MATEMÁTICA, SABERES OBJETIVADOS E A CONSTITUIÇÃO DE UMA DISCIPLINA ${ }^{\alpha}$
}

\author{
History of mathematics education, objectified knowledge and the \\ constitution of a discipline
}

\section{Historia de la educación matemática, saberes objetivados y la constitución de una disciplina}

\section{Maria Cristina Araújo de Oliveira ${ }^{\beta}$}

Data de recepção: 18/03/2019 • Data de aceitação: 23/07/2019

Resumo. Objetiva-se discutir o papel da História da educação matemática (HEM) na formação de professores que ensinam matemática. Argumenta-se pela inserção desse saber como uma disciplina em cursos de graduação e pós-graduação. Nessa direção pretende-se mostrar a relevância da exploração, em nível de ensino, dos conhecimentos já produzidos no domínio da pesquisa em história da educação matemática para a formação do professor que ensina matemática. De maneira geral pode-se dizer que a pesquisa nesse campo se orienta por questões próximas das práticas profissionais dos professores, que podem ser desnaturalizadas se examinadas do ponto de vista histórico. Tornar acessível aos professores em formação os resultados dos estudos produzidos sobre a HEM sob a forma de disciplina cria a possibilidade de imbricamento do ensino com a pesquisa de maneira a

\footnotetext{
${ }^{a}$ Este artigo dá continuidade e aprofunda outros dois que discutem a importância da História da educação matemática (HEM) como uma disciplina na formação de professores que ensinam matemática: Maria Cristina Araújo de Oliveira, «A História da educação matemática como disciplina na formação de professores que ensinam Matemática», Cadernos de História da Educação 16 (2017): 653-665 e Maria Cristina Araújo de Oliveira, «História da educação matemática contributos para a formação de professores que ensinam Matemática», Em Actas IV Congresso Iberoamericano de História da Educação Matemática, editado por Dolores Carrillo Gallego et al., 52-59 (Murcia: Universidade de Murcia, 2018).

${ }^{\beta}$ Departamento de Matemática e Programa de Pós-Graduação em Educação Matemática, Universidade Federal de Juiz de Fora, Juiz de Fora, Brasil. mcrisoliveira6@gmail.com (iD http://orcid.org/0000-0003$3346-1578$
}

Como citar este artigo: Oliveira, Maria Cristina Araújo de. «História da educação matemática, saberes objetivados e a constituição de uma disciplina». Historia y Memoria de la Educación 11 
garantir um aporte diferenciado sobre a matemática escolar e fomentar novas questões de investigação.

Palavras-chave: História da educação matemática; Disciplina acadêmica; Formação de professores.

Resumen. Se propone discutir el papel de la historia de la educación matemática (HEM) en la formación de profesores que enseñan matemática. Se estudia la inserción de este saber como una disciplina en cursos de pregrado y posgrado. En esta dirección, se pretende mostrar la relevancia de la exploración, en un nivel de enseñanza, de los conocimientos ya producidos en el dominio de la investigación en historia de la educación matemática para la formación del profesor que enseña matemática. De manera general se puede decir que la investigación en este campo se orienta por preguntas cercanas a las prácticas profesionales de los profesores en formación los resultados de los estudios producidos sobre la HEM bajo la forma de disciplina crea la posibilidad de fusionar la enseñanza con la investigación de manera que garantiza un aporte diferente sobre la matemática escolar y fomenta nuevas preguntas de investigación.

Palabras-clave: Historia de la educación matemática; Disciplina académica; Formación de profesores.

Abstract. The purpose of this paper is to discuss the role of the History of mathematics education (HEM) in the training of teachers who teach mathematics. We propose the insertion of this specialized knowledge as a subject in undergraduate and postgraduate courses. In this sense we intend to show the relevance of the exploration, at the teaching level, of the knowledge previously produced in the field of research in the history of mathematics education for the training of teachers in this subject. In general, it can be said that research in this field is guided by issues close to the professional practices of teachers, which can be denatured if examined from a historical point of view. By giving the results of studies produced on HEM the form of a discipline accessible to the teachers in training, we believe we can create the possibility of linking teaching with research in a way that ensures a differentiated contribution to school mathematics and fosters new research questions.

Keywords: History of mathematics education; Academic discipline; Teacher training.

\section{INTRODUÇÃO}

A temática da inclusão ou da participação da História da Educação Matemática (HEM) na formação de professores que ensinam matemática esteve presente nos dois últimos Encontros Nacionais de História 
da Educação Matemática (ENAPHEM), realizados em 2016 e 2018, no Brasil.

Intenta-se neste texto discutir o papel de saberes objetivados e da criação da disciplina História da educação matemática ${ }^{1}$ no âmbito da formação inicial de professores de matemática.

Nessa direção pretende-se mostrar a relevância da exploração, em nível de ensino, dos conhecimentos já produzidos no domínio da pesquisa em HEM para a formação do professor que ensina matemática.

\section{SABERES OBJETIVADOS E A FORMAÇÃO DE PROFESSORES QUE ENSINAM MATEMÁTICA}

Em texto recente, de 2019, Valente aborda a noção de saber objetivado de modo a construir uma conceituação a partir de abordagens mais amplas sobre saberes e epistemologia, tomando para tanto escritos de diversos autores, em especial capítulos do livro Épistémologie, savoirs et champs disciplinaires: questions d'apprentissage, organizado por Marin \& Savatovsky. ${ }^{2}$

Tomando como referência o capítulo elaborado por Cusset, que aponta para uma dicotomia entre a reflexão pedagógica e a epistemológica, restrita aos cursos de filosofia, na formação de professores franceses, Valente sintetiza que os saberes profissionais estão «alijados dos campos científicos consagrados e não ganham lugar na discussão epistemológica». ${ }^{3}$ Para Cusset tal discussão pode trazer contribuições para a formação de professores na medida em que possam

\footnotetext{
${ }_{1}$ Acompanhamos a distinção proposta por Wagner Rodrigues Valente en «História da educação matemática: considerações sobre suas potencialidades na formação do professor de matemática», Bolema 23 (2010): 123-136, pela qual utilizaremos educação matemática para nos referirmos aos processos educativos relativos ao ensino e à aprendizagem de Matemática ao longo dos tempos, diferenciando da Educação Matemática, que no Brasil, corresponde a um campo de investigação que emergiu a partir da década de 1980, tendo como referência a criação da Sociedade Brasileira de Educação Matemática em 1988.

2 Brigitte Marin \& Dan Savatovsky (dir.), Épistémologie, savoirs et champs disciplinaires: questions d'apprentissage (Créteil: Université Paris-Est Créteil. no. 4, 2013), 103-112.

${ }^{3}$ Wagner Rodrigues Valente, «Saber objetivado e formação de professores: reflexões pedagógico-epistemológicas», História da Educação 23 (2019): 6.
} 
considerar a articulação, tanto do ponto de vista histórico como metodológico e cognitivo, entre paradigmas científicos e modelos de aprendizagem, entre instituição e desenvolvimento de uma ciência e delimitação de uma disciplina escolar, entre formas de transmissão e vulgarização científica [...]. ${ }^{4}$

Outro capítulo da mesma obra, escrito por Bernard, na mesma direção, defende que um ensino de história e de epistemologia das disciplinas escolares nos cursos de formação de professores possibilitaria uma «forma de reflexão verdadeiramente útil para a prática e para o seu desenvolvimento». ${ }^{5}$ Em síntese, Valente aponta que ambos os textos colocam em evidência «a necessidade de formar o professor por meio de uma discussão epistemológica que coloque em cena as matérias e disciplinas escolares». ${ }^{6}$

Nesse mesmo texto de Valente, o autor retoma um artigo dele de 2013, mesmo ano da publicação anteriormente referenciada, em que defende a importância na formação do professor que ensina matemática do estudo histórico da matemática escolar. Tal estudo traria sentido aos conteúdos que são ensinados na educação básica, possibilitando explicar a ausência ou a permanência de temas no ensino de matemática.

Em síntese, Valente defende que

Desse modo, eleger como tema de discussão as disciplinas escolares na formação de professores coloca a instituição escolar e o trabalho docente noutro patamar em termos dos saberes. Afasta a ideia de que a escola é lugar submisso de transmissão de saberes elaborados fora dela. Dá sentido, assim, ao debate epistemológico tratado no âmbito do ensino, via formação de professores. O tema, dessa forma, não será monopólio da filosofia, voltado tão somente para as disciplinas científicas universitárias, classicamente já estabelecidas. [...] Nessa problematização caberia analisar como o modo disciplinar organiza, sistematiza os saberes. E como isso ocorre, também, no meio escolar. Como as disciplinas

\footnotetext{
${ }^{4}$ Cusset apud Rodrigues Valente, «Saber objetivado e formação de professores», 7.

5 Bernard apud Rodrigues Valente, «Saber objetivado e formação de professores», 7.

6 Rodrigues Valente, «Saber objetivado e formação de professores», 8.
} 
objetivam os saberes, dão a eles a possibilidade de que sejam comunicáveis, transmissíveis, objetos e ferramentas a estarem presentes na formação de professores e também no ensino escolar.7

Com esta elaboração, Valente mais uma vez evidencia as possibilidades que o estudo histórico da matemática escolar traz para a formação dos professores que ensinam matemática. Mas quais os meios, ou ainda de que forma, os futuros professores podem fazer, ou ter acesso a estes estudos históricos? A nosso ver, esta é uma das finalidades da História da educação matemática enquanto campo de investigação, produzir trabalhos ou criar meios que permitam aos futuros professores conhecer e se apropriar da matemática escolar numa perspectiva histórica.

O surgimento da escola como um espaço de aprendizagens intencionais, sem necessariamente buscar uma aplicação prática voltada para o mundo do trabalho, criou uma distinção entre saber e saber fazer; neste contexto, o saber objetivado torna-se um pleonasmo. Citando Vicent, Valente explica a afirmação, "A pedagogia [...] se articula a um modelo explícito, objetivado e fixo de saber a transmitir». A escola tem como objetivo fazer com que os alunos aprendam saberes que resultam de um «trabalho de classificação, divisão, articulação, estabelecimento de relações, comparação, hierarquização etc.», ${ }^{8}$ sistematizados pela escrita.

Os recentes estudos em torno dos saberes da ação para a formação de professores conferem sentido ao termo saberes objetivados. Recorre-se aqui a Barbier para a conceituação do termo:

o campo dos saberes objetivados pertence à mesma zona semântica da cultura, das regras, dos valores. Esta zona refere-se provavelmente a realidades que têm o estatuto de representações ou de sistemas de representações dando lugar a enunciados proposicionais e tendo por objeto uma valorização social sancionada por uma atividade de transmissão-comunicação. Essas realidades distinguem-se daqueles que as enunciam ou daqueles que delas se apropriam. Elas são conserváveis, cumulativas e apropriáveis. No âmbito dessa zona semântica, os saberes objetivados podem

\footnotetext{
7 Rodrigues Valente, «Saber objetivado e formação de professores», 9.

${ }^{8}$ Vicent et al. apud Rodrigues Valente, «Saber objetivado e formação de professores», 11.
} 
ser definidos como enunciados de proposições sendo objeto de um julgamento social que se situa no registro da verdade ou da eficiência. ${ }^{9}$

Assumindo a perspectiva da importância dos saberes objetivados, aqueles que são segundo os autores anteriormente mencionados «conserváveis, cumulativos e apropriáveis», para a formação dos professores que ensinam matemática é que procuramos situar a História da educação matemática e sua finalidade maior.

\section{SABERES OBJETIVADOS E A HISTÓRIA DA EDUCAÇÃO MATEMÁTICA}

Alguns estudos históricos analisam as relações entre as matérias escolares e os campos disciplinares em nível do ensino superior. Os estudos sobre as matérias escolares, segundo Goodson, "advêm de duas perspectivas principais, a sociológica e a filosófica». ${ }^{10}$ Mas o autor destaca outros pesquisadores, Bourdieu e Young, por exemplo, que desenvolvem e defendem abordagens históricas para melhor compreender e para propor reformulações e novas proposições curriculares. Goodson considera que «o exame do processo pelo qual se cria uma matéria escolar deve gerar insights históricos úteis». ${ }^{11}$

O pesquisador analisa a relação entre as matérias escolares e as disciplinas que supostamente se relacionam a elas em nível superior. Conclui que a autonomia ou a dependência entre essas modalidades de ensino varia de acordo com o estágio de evolução das matérias.

Um estudo realizado com a matéria ciências no Reino Unido, a partir do século XIX, sugere um modelo de desenvolvimento da matéria escolar compreendido por três estágios. Num primeiro, os argumentos de pertinência e utilidade garantem a inserção da matéria no currículo escolar. Nessa etapa os professores raramente são especialistas, mas sim entusiastas da inserção curricular. "O critério dominante é a relevância

\footnotetext{
9 Barbier apud Rodrigues Valente, «Saber objetivado e formação de professores», 13.

${ }^{10}$ Ivor Goodson, «Tornando-se uma matéria acadêmica: padrões de explicação e evolução», Teoria e Educação 2 (1990): 230.

${ }^{11}$ Goodson, «Tornando-se uma matéria acadêmica», 232.
} 
para as necessidades e interesses dos aprendizes».12 Num estágio intermediário, emerge uma tradição de trabalho acadêmico e um corpo de especialistas que são recrutados como professores. Nessa etapa tem peso tanto a relação com os problemas e interesses dos estudantes como a reputação e o status acadêmico do conhecimento. O processo de institucionalização crescente da matéria interfere na seleção e organização do seu conteúdo. No estágio final, "prelúdio do desencantamento»,13 os professores são especialistas, a seleção dos conteúdos da matéria é, em grande medida, determinada pelos pesquisadores da área, prevalecendo as referências do meio acadêmico. "Os estudantes são iniciados numa tradição, suas atitudes aproximam-se da passividade e da resignação».14

O modelo anteriormente descrito não pressupõe uma explicação monolítica da relação entre uma matéria e uma disciplina. O exame do processo histórico de criação de uma matéria escolar permite compreender o constante fluxo entre as matérias e as disciplinas.

O estudo histórico da criação da matéria escolar Geografia indica como a mesma impulsionou a criação de uma disciplina acadêmica. A consolidação como matéria escolar e a criação da Geographical Association, em 1893, tiveram papel determinante, segundo Goodson, na culminância da área de conhecimento da Geografia em nível superior no Reino Unido.

A inclusão da Geografia nos exames escolares nos diferentes níveis e a elaboração dos mesmos sob a responsabilidade de professores especializados em Geografia reforçava a necessidade de formação em nível superior de tais especialistas.

No caso da História da educação matemática como uma disciplina têm-se uma situação diferente na medida em que, em princípio, se associa ao processo de formação dos professores que ensinam matemática em sua dimensão profissional, ou seja, não necessariamente se situa no campo dos saberes a ensinar.

\footnotetext{
12 Goodson, «Tornando-se uma matéria acadêmica», 235.

13 Goodson, «Tornando-se uma matéria acadêmica», 236.

14 Goodson, «Tornando-se uma matéria acadêmica», 236.
} 
A obra organizada por Jean Boutier, Jean-Claude Passeron e Jacques Revel, Qu'est-ce qu'une discipline?, publicada em 2006, dedica-se a problematizar a noção de disciplina a partir dos campos da antropologia, da sociologia e da história. Na apresentação, os organizadores apontam para a complexidade da proposta anunciando que a resposta ao que é uma disciplina, longe de ser meramente classificatória, é o trabalho do historiador e do sociólogo para «descrever a identidade social, a identidade profissional ou a identidade epistemológica de diferentes corpus de saberes especializados, reconhecidos e transmitidos como tais pelas organizações universitárias na nossa sociedade».15

O sistema disciplinar que faz «coexistir uma organização do mundo sábio, uma pedagogia eficaz de transmissão e uma renovação contínua da possibilidade de descoberta, se encontra profundamente perturbado». ${ }^{16}$ Esta perturbação decorre não só das mudanças das funções sociais da ciência, mas também da trajetória das problemáticas de pesquisa cada vez mais especializadas e, contraditoriamente, da própria emergência dos paradigmas trans ou interdisciplinares.

No primeiro capítulo desta obra, Fabiani ${ }^{17}$ dedica-se a problematizar a que serve a noção de disciplina. Para ele disciplina e ciência são equivalentes para designar o conjunto de relações entre objetos e pessoas que tem a especificidade de um domínio de saber ou de um programa de pesquisa. A disciplina é a cristalização e a estabilização de uma prática científica bem como modo de ocupação reconhecido de uma configuração mais ampla advinda da ciência.

A disciplina traduz da atividade científica uma forma particular de divisão do trabalho no mundo social. Ela apresenta evidências palpáveis da organização cotidiana da pesquisa para o ensino. Se por um lado permite decompor um saber científico de maneira coerente e complementar de modo a possibilitar o conhecimento, por outro esta

\footnotetext{
15 Jean Boutier, Jean-Claude Passeron \& Jacques Revel (eds.), Qu'est-ce qu'une discipline? (Paris: École des Hautes Études en Sciences Sociales, 2006), 7.

16 Boutier, Passeron \& Revel, Qu'est-ce qu'une discipline?, 7.

17 Jean-Louis Fabiani, «À quoi sert la notion de discipline?», em Qu'est-ce qu'une discipline?, eds. Jean Boutier, Jean-Claude Passeron \& Jacques Revel (Paris: École des Hautes Études en Sciences Sociales, 2006), 11-34.
} 
configuração traz limitações na medida em que tenta descrever uma maneira eficaz de operar sobre atividade de conhecer.

Duas perspectivas históricas muito diferentes dizem respeito à disciplina. A primeira, anterior à ciência moderna, compreende as dimensões da relação pedagógica a partir do aluno, do discípulo. Tem como finalidade colocar o aluno no centro do sistema e organizar o saber a partir de um objetivo pedagógico; mais que um sujeito conhecedor são as formas de controle pedagógico que ocupam uma posição central na disciplina. A segunda perspectiva é a organização particular pela qual se desenvolvem os saberes modernos como conjuntos de práticas codificadas e reconhecidamente válidas por um coletivo delimitado. A coexistência destas duas perspectivas de disciplina coloca em tensão as necessidades da comunicação pedagógica com a exigência de inovação. Por um lado, são definidas as condições da reprodução de um corpo de conhecimentos por meio da fidelidade dos exercícios. Por outro, a exigência de progresso científico estimula o horizonte da pesquisa.

Segundo Fabiani, um entendimento equivocado sobre a revolução científica de Khun é pensar que é a inovação que move a ciência; ao contrário, o que mostra é que a situação tradicionalista da atividade científica cotidiana é que permite introduzir a mudança na tradição. A inovação se relaciona à existência de comunidades disciplinares. $\mathrm{Na}$ verdade, ela tem lugar por um aprofundamento da tradição; como um paradoxo que não é evidente, o pesquisador que não adere ao quadro normal da ciência é menos suscetível de produzir inovação.

A estrutura disciplinar coloca em tensão a tradição por meio das disciplinas e a possibilidade de inovação científica. Para Fabiani, a disciplina não é inteiramente redutível ao saber ou a uma ciência na medida em que ela é indissociável de um sistema de ensino particular. A definição mais geral de uma disciplina pode ser enunciada como um corpo de conhecimento inscrito em textos, exemplos paradigmáticos e formas de instrumentação que são objeto de uma transmissão pedagógica que necessita ser colocada em ação ou conformada. Em síntese, pode-se dizer que uma disciplina é uma estabilização de um objeto de conhecimento com fronteira segura e com o estabelecimento de modos unificados de tratamento dos objetos previamente decompostos. 
Os estudos de Hofstetter e Schneuwly sobre o processo de disciplinarização das ciências da educação e das didáticas das disciplinas, enquanto novos campos disciplinares na Europa, mostram a convergência em quatro aspectos:

Uma disciplina emerge e se desdobra via a conquista de uma base institucional permitindo a profissionalização da pesquisa, que se concretiza pela institucionalização de postos, cadeiras, laboratórios, centros, garantindo a criação de um corpo de profissionais especializados no domínio.

Esta base institucional favorece a constituição de redes de comunicação, tais como de associações de pesquisadores, de manifestações científicas (congressos, seminários etc.) e, sobretudo, de suportes editoriais (especialmente revistas) que permitem a construção de uma comunidade de cientistas trabalhando em torno das mesmas problemáticas.

Esta infraestrutura institucional e comunicacional condiciona a renovação dos conhecimentos, instituindo o campo como empreendimento coletivo de pesquisa; esta produção de conhecimentos passa pela elaboração e aperfeiçoamento contínuo de conceitos e modelos teóricos, bem como das metodologias de coleta e análise de dados no domínio, respondendo aos critérios de legitimidade científica dos quais depende o reconhecimento social e científico da disciplina.

Estas dimensões permitem uma socialização no campo e ela ocorre tanto mais facilmente quanto a disciplina se implanta como uma unidade de estreito imbricamento de ensino e pesquisa. Este desafio formativo está relacionado com aquele da capacidade de uma disciplina de determinar ela mesma os critérios de legitimidade de sua reprodução e de formar seus representantes. ${ }^{18}$

Refletindo sobre a criação de uma base institucional que permita a profissionalização da pesquisa em HEM, o que se verifica é que, em nível

\footnotetext{
18 Rita Hofstetter \& Bernard Schneuwly, «Disciplinarisation et disciplination consubstantiellement liées. Deux exemples prototypiques sous la loupe: les sciences de l'éducation et des didactiques des disciplines», em Disziplin-Discipline, ed. Balz Engler (Fribourg: Academic Press, 2014), 28-29.
} 
de pós-graduação, diferentes Programas na área de Ensino de Ciências e Matemática e de Educação já incluem pesquisadores que vêm atuando nessa temática e formando novos pesquisadores. Poderíamos dizer, a tomar como referência somente os pesquisadores que integram o Grupo de Pesquisa de História da Educação Matemática no Brasil (GHEMAT), que, em todas as cinco regiões do país, com maior ou menor intensidade esse quadro se verifica. Em grande medida, pode-se avaliar que um corpo de profissionais especializados nesse domínio vem se formando.

Em termos da constituição de redes de comunicação, a área de História da educação matemática vem se organizando de maneira bastante efetiva com a realização de encontros nacionais e internacionais e a publicação de periódicos. Os encontros internacionais mais expressivos são o Congresso Iberoamericano de História da Educação Matemática, que teve início em 2011 e é realizado a cada dois anos, e a International Conference on the History of Mathematics Education, a partir de 2009, com periodicidade bianual. O evento brasileiro mais representativo é o Encontro Nacional de Pesquisas em História da Educação Matemática, cuja periodicidade é bianual.

Quanto aos periódicos específicos da temática, circulou entre 2006 e 2016 o International Journal for the History of Mathematics Education, e atualmente a Revista de História da Educação Matemática - HISTE$M A T^{19}$ é publicada quadrimestralmente.

Cabe ainda mencionar um espaço importante para a discussão sobre a renovação dos conhecimentos, a elaboração e o aperfeiçoamento da discussão teórica e metodológica da História da educação matemática que é a criação de um grupo de trabalho específico de Hem no âmbito da Sociedade Brasileira de Educação Matemática (SBEM). Esse grupo se articula a diversas iniciativas promovidas pela SBEM, tais como o Seminário Internacional de Pesquisa em Educação Matemática (SIPEM).

Talvez seja possível avaliar que o embricamento entre ensino e pesquisa é ainda um aspecto da área de História da educação matemática que merece maior atenção, sobretudo em nível dos cursos de formação de professores que ensinam matemática.

${ }_{19}$ Disponível em http://histemat.com.br/index.php/HISTEMAT. 


\section{A HISTÓRIA DA EDUCAÇÃO MATEMÁTICA NA FORMAÇÃO DOS PROFESSORES QUE ENSINAM MATEMÁTICA}

Os conhecimentos já produzidos no campo da História da educação matemática no Brasil abrangem múltiplas temáticas considerando sua relação com a matemática - estudam temas específicos como geometria ou aritmética; bem como níveis de ensino. Fato histórico importante constitui a criação da disciplina matemática na educação básica. Essa disciplina é resultado de um movimento internacional de renovação do ensino de matemática, que tem como marco importante a criação da International Commission on Mathematical Instruction, em 1908, presidida pelo matemático alemão Félix Klein. As apropriações brasileiras da discussão internacional foram sistematizadas principalmente pelo educador matemático Euclides Roxo, que esteve à frente da formulação e proposição dos currículos de Matemática e da produção de livros didáticos dessa disciplina no Brasil a partir da década de 1920.

Podemos exemplificar alguns estudos já realizados que trazem resultados importantes para a formação do futuro professor. Trabalhos que mostram como determinados assuntos matemáticos se consolidaram ou se reconfiguraram no currículo com o Movimento da Matemática Moderna, ${ }^{20}$ por exemplo, contribuem no processo de desnaturalização dos currículos e das matérias de ensino.

A partir das propostas da Matemática Moderna o estudo de matrizes se firmou no ensino colegial ${ }^{21}$, conforme o trabalho de Lopes $^{22}$ e se mantém até a atualidade em grande parte das instituições que oferecem o ensino médio, denominação atual para o colegial.

\footnotetext{
${ }^{20}$ Em nível internacional o Movimento se iniciou na década de 1950, as propostas incluíam modernizar o ensino de Matemática acompanhando o desenvolvimento científico do campo de investigação. A característica de unidade da Matemática se consolidaria pelas estruturas algébricas, o que colocou em destaque a teoria dos conjuntos. No Brasil, o MMM se fortalece e consolida na década de 1960.

${ }^{21}$ Correspondente aos 3 últimos anos do ensino secundários, estudantes de 15 a 18 anos.

22 Marcelo dos Reis Lopes, «Matrizes: história de um conteúdo escolar» (Dissertação de Mestrado em Programa de Pós-Graduação em Ensino de Matemática, Universidade Federal do Rio de Janeiro, 2012).
} 
A noção de função assumiu uma abordagem conjuntista respondendo à defesa da unidade matemática proposta por esse Movimento. ${ }^{23}$ Com isso, a representação por meio de diagramas passou a ocupar grande parte do tempo e das atividades dedicadas ao ensino de função, enquanto o enfoque da relação de dependência entre as variáveis deixou de ser enfatizado.

Ou ainda, diferentes abordagens geométricas foram incentivadas e exploradas a partir da referência da Matemática Moderna pela qual a ênfase estava nas estruturas algébricas. Cabe mencionar a introdução da geometria por transformações, a retomada da geometria analítica. ${ }^{24}$

Para os professores que ensinam matemática para as crianças, é relevante conhecer as transformações pelas quais os saberes elementares matemáticos passaram ao longo do tempo. A própria constituição de uma matemática a ensinar, ou seja, fruto da elaboração para a atividade de ensino, é objeto de pesquisas mais recentes organizadas em torno de um projeto temático, coordenado por Wagner Valente, sobre a matemática como um saber profissional do professor. Os primeiros resultados apontam para uma mudança importante na formação dos professores primários, que se inicia a partir do final do século XIX, quando se dá início à caracterização de uma matemática para ensinar, que compreende metodologias, materiais didáticos, sequências para o ensino. ${ }^{25} \mathrm{No}$ Brasil, este processo ocorre no momento da circulação do método intuitivo, ${ }^{26}$

\footnotetext{
${ }^{23}$ Alexandre Souza de Oliveira, «A abordagem do conceito de função em livros didáticos ginasiais: uma análise em tempos modernos (décadas de 1960 a 1970)» (Dissertação de Mestrado em Educação Matemática, Universidade Bandeirante de São Paulo, 2009).

${ }_{24}$ Maria Célia Leme da Silva, «A geometria escolar e o Movimento da Matemática Moderna: em busca de uma nova representação», em A Matemática Moderna nas escolas do Brasil e de Portugal: contribuição para a história da educação matemática, eds. Cláudia Flores \& Joseane Pinto de Arruda (São Paulo: Annablume, vol. 1, 2010), 65-88; Josélio Lopes Valentim Júnior, «A geometria analítica como conteúdo do ensino secundário: análise dos livros didáticos utilizados entre a Reforma Capanema e o MMM» (Dissertação de Mestrado em Educação Matemática, Universidade Federal de Juiz de Fora, 2013).
}

${ }_{25}$ Wagner Rodrigues Valente, «O saber profissional do professor que ensina matemática: história da matemática a ensinar e da matemática para ensinar em construção», em História da Educação Matemática e Formação de professores, orgs. Bruno Alves Dassiê \& David Antônio da Costa (São Paulo: LF editorial, vol. 1, 2018), 49-84.

${ }^{26}$ O método intuitivo marcou o processo de estruturação da instrução pública no Brasil de meados do século XIX às primeiras décadas do século XX. Sua adoção orientou as proposições oficiais e outros impressos que se ocuparam da educação nacional no período. Com base em Pestalozzi a educação elementar repousaria sobre a tríade "cabeça, coração e mão», que está associada a três formas de atividades fundamentais: conhecer, querer e poder. 
que coincide com o surgimento dos grupos escolares ${ }^{27}$ e da escola graduada.

Sobre a aritmética, duas teses de doutorado produzidas em 2017 abordam o tema utilizando a matemática a ensinar e para ensinar como categorias históricas de análises. ${ }^{28}$ As pesquisas de Oliveira e Pinheiro ${ }^{29}$ caracterizam uma aritmética intuitiva e uma aritmética sob medida, respectivamente. A aritmética intuitiva possibilitou que os saberes matemáticos compreendidos relacionassem a vida social da criança com a vida escolar. «Desta forma, Aritmética passou a ser uma matéria que dava oportunidade da criança aplicar na vida social aquilo que aprendia na vida escolar. Uma matéria que extraía da vida social as situações para compor e conduzir as tarefas da vida escolar».30

A aritmética sob medida, elaborada pela ordem psicológica e dosada segundo a maturidade infantil —amparada em testes estatísticos fundamentados na psicologia experimental, reorganizou o estudo das operações. Em lugar de aprender a adição, depois a subtração, em seguida a multiplicação e por último a divisão, como operações separadas, os estudos experimentais mostraram que era mais fácil para a criança estudar a multiplicação após a adição, como uma sequência de operações aditivas. De modo análogo, a divisão seria então tratada após a subtração, também como sequência de subtrações. ${ }^{31}$

A geometria a ensinar entre a última década do século XIX e as primeiras décadas do XX tomou como pressuposto a aprendizagem por meio dos sentidos, e pela perspectiva pestalozziana de conhecimento elementar dada pela terna forma, número e palavra; deveria pautar-se pela observação, manipulação, comparação e descrição das formas.

\footnotetext{
${ }_{27} \mathrm{O}$ modelo dos grupos escolares se apoiava no princípio da seriação e conferia destaque aos métodos pedagógicos, especialmente ao método intuitivo. Segundo Diana Gonçalves Vidal, Grupos escolares: cultura escolar primária e escolarização da infância no Brasil (1893-1971) (Campinas, SP: Mercado das Letras, 2006), os grupos escolares consolidaram no país a representação do ideal da escola pública elementar.

${ }^{28}$ Rodrigues Valente, «O saber profissional do professor que ensina matemática».

29 Marcus Aldenisson de Oliveira, «Mudanças nos saberes elementares matemáticos dos anos iniciais: circulação e apropriação do método intuitivo para o ensino de Aritmética (1879-1960)» (Tese de Doutorado em Programa de Pós-Graduação em Educação e Saúde, Universidade Federal de São Paulo, 2017); Nara Vilma Lima Pinheiro, «A aritmética sob medida: a matemática em tempos da pedagogia científica» (Tese de Doutorado em Programa de Pós-Graduação em Educação e Saúde, Universidade Federal de São Paulo, 2017).

30 Aldenisson de Oliveira, «Mudanças nos saberes elementares matemáticos dos anos iniciais», 245.

${ }^{31}$ Lima Pinheiro, «A aritmética sob medida: a matemática em tempos da pedagogia científica».
} 
O aluno deveria ter uma sensação agradável ao envolver a «bola» em suas mãos enquanto que o mesmo não aconteceria com o cubo. Só então a partir dessa sensação e da observação de que a «bola» rola e o cubo não, é que o sólido seria nomeado por esfera, algum tempo deveria ser gasto até que a palavra fosse bem pronunciada pelos alunos. ${ }^{32}$

Os estudos já realizados sobre a geometria a ensinar mostram a força da referência euclidiana na cultura escolar, mesmo com a tentativa de inserir e transformar esta abordagem, durante o MMM. ${ }^{33}$ É possível pensar dessa forma na adequação deste saber como introdutório do pensamento geométrico para as crianças. Cabe ressaltar a constituição de materiais didáticos não só para observação, mas para manipulação, montagem, etc. que ao longo do tempo contribuíram na elaboração de uma geometria para ensinar.

No final do século XIX e nas primeiras décadas do século XX, o desenho enquanto saber teve função importantíssima na formação dos professores primários e no desenvolvimento das crianças. ${ }^{34}$

Desde auxiliar na aprendizagem das letras do alfabeto, passando por uma metodologia para ensinar conteúdos a recurso utilizado para expressar conhecimentos adquiridos pelos alunos. A partir da década de 1960 nota-se a ausência do Desenho de forma explícita. Cabem outras investigações que permitam identificar a transformação/incorporação do Desenho em rubricas como Educação Artística, Artes Aplicadas ou Desenho Geométrico. ${ }^{35}$

O conceito de número visto como um dos primeiros saberes a ser ensinado às crianças desde os tempos mais antigos da escolarização brasileira

\footnotetext{
32 Maria Cristina Araújo de Oliveira, «A geometria como disciplina do curso de formação de professores primários: a influência do método intuitivo nas primeiras décadas do século XX no Brasil», Perspectiva 34 (2016): 113.

${ }_{3}$ Maria Cristina Araújo de Oliveira, "Percurso investigativo sobre a geometria e o desenho como saberes profissionais de professores dos anos iniciais no Brasil», Paradigma 39 (2018): 175-189.

34 Referência representativa do conhecimento produzido sobre esse tema é a publicação do número especial sobre a História do ensino de geometria e de desenho da Revista de História da Educação Matemática - HISTEMAT 2, no. 2 (2016).

35 Araújo de Oliveira, "Percurso investigativo sobre a geometria e o desenho como saberes profissionais de professores dos anos iniciais no Brasil», 187.
} 
passou a exigir como pré-requisito o estudo de conjuntos, quando a visão da teoria dos conjuntos como elemento de unidade na Matemática Moderna se dissemina a partir da década de $1960 .{ }^{36}$

Algumas pesquisas sobre a história de disciplinas acadêmicas do curso de licenciatura em Matemática mostram os processos de reconfiguração da História da Matemática e da Prática de Ensino de Matemática, ao passo que se identifica «uma cultura de reprovação e de estabilidade de conteúdos nas disciplinas Cálculo Diferencial e Integral e Geometria Analítica, rubricas que, se pode dizer, integram um 'núcleo duro' dos cursos de Ciências Exatas que ainda demandam propostas e ações por parte da comunidade de educadores matemáticos».37

Uma perspectiva ainda pouco explorada, mas defendida frequentemente por Valente, ${ }^{38}$ é a utilização da História da educação matemática como metodologia para o trabalho com a matemática escolar no âmbito da formação dos professores.

Não é intenção deste texto esgotar as possibilidades de propostas que podem ser levadas aos cursos de formação de professores que ensinam matemática por meio de textos e atividades que mobilizem a História da educação matemática. Ao contrário, apresenta-se aqui um reduzido número de pesquisas para dar visibilidade a conhecimentos produzidos na área para a formação dos professores que ensinam Matemática.

\section{ALGUMAS EXPERIÊNCIAS COM A HISTÓRIA DA EDUCAÇÃO MATEMÁTICA}

Pode-se dizer que a História da educação matemática está em processo de disciplinarização tanto na graduação nos cursos de licenciatura como na pós-graduação em Programas em Educação Matemática. A

\footnotetext{
${ }^{36}$ Maria Célia Leme da Silva \& Wagner Rodrigues Valente, «Uma breve história do ensinar e aprender matemática nos anos iniciais: uma contribuição para a formação de professores», Educação Matemática Pesquisa 15 (2013): 837-855.

37 Maria Cristina Araújo de Oliveira, «O que pesquisas históricas sobre disciplinas de cursos de licenciatura em Matemática brasileiros revelam sobre essa formação?», Jornal Internacional de Estudos em Educação Matemática 6 (2013): 164.

38 Wagner Rodrigues Valente, "O Lugar da Matemática Escolar na Licenciatura em Matemática», Bolema 27 (2013): 939-955.
} 
seguir apresentam-se algumas experiências com essa disciplina em diferentes instituições do Brasil.

Ao que tudo indica a disciplina de História da educação matemática foi inserida no curso de licenciatura em Matemática de forma pioneira na Universidade Federal do Rio Grande do Norte (UFRN) a partir de 2001.39 A disciplina trata historicamente o ensino da matemática desde a Antiguidade passando pelos dois movimentos internacionais de modernização do ensino de matemática, finalizando com o surgimento do campo da Educação Matemática. A bibliografia é composta por:

- Miorim, Maria Angela. Introdução à História da Matemática. São Paulo: Ed. Da Unicamp, 1992.

- Silva, Circe Mary Silva da. Matemática positivista e sua difusão no Brasil. Vitória: Edufes, 1999.

- Silva, Clóvis Pereira da. A Matemática no Brasil. Uma história do seu desenvolvimento. Curitiba: Edufpr, 1992.

- Valente, Wagner Rodrigues. Euclides Roxo e a modernização do ensino de Matemática no Brasil. Brasília: Editora da UNB, 2004.

- Valente, Wagner Rodrigues. O nascimento da Matemática do ginásio. São Paulo: Annablume, 2004.

- Valente, Wagner Rodrigues. Uma história da Matemática escolar no Brasil (1730-1930). São Paulo: Annablume, 1999.

A História da educação matemática foi inserida na História da Matemática ${ }^{40}$ enquanto disciplina da licenciatura em Matemática na Universidade Federal de Juiz de Fora (UFJF) a partir de 2009. Os temas abordados compreendem o estudo da forma escolar da matemática no Brasil desde os tempos de colônia e os dois movimentos internacionais de renovação do ensino de matemática que são analisados tanto em perspectiva internacional quanto em suas apropriações nacionais. Da bibliografia indicada fazem parte:

\footnotetext{
39 Iran Abreu Mendes, «História da educação matemática na formação de professores de Matemática: uma experiência disciplinar na UFRN», Revista de História da Educação Matemática 2 (2016): 185-199.

40 Disciplina obrigatória com 4 créditos.
} 
- Guimarães, Henrique Manuel «Por uma Matemática nova nas escolas secundárias - perspectivas e orientações curriculares da Matemática Moderna». Em A Matemática Moderna nas escolas do Brasil e de Portugal: primeiros estudos, organizado por José Manuel Matos \& Wagner Rodrigues Valente, 21-45. São Paulo: Da Vinci, 2007.

- Miorim, Maria Angela. Introdução à história da educação matemática. São Paulo: Atual, 1998.

- Oliveira, Maria Cristina Araújo de, et al. O Movimento da Matemática Moderna - história de uma revolução curricular. Juiz de Fora: Editora UFJF, 2011.

- Valente, Wagner Rodrigues. Uma história da matemática escolar no Brasil, 1730-1930. São Paulo: Editora Annablume/Fapesp, 1999.

- Valente, Wagner Rodrigues. Euclides Roxo e a modernização do ensino da matemática no Brasil. Brasília: Editora da Universidade de Brasília, ed. 2, v. 1, 2004.

Quando da inserção da História da educação matemática na disciplina de História da Matemática da UFJF uma das dificuldades à época foi formular um elenco de temas a serem discutidos de modo que associados a eles uma bibliografia pudesse ser disponibilizada aos alunos. A produção de bibliografia acessível e adequada para os estudantes de graduação é um dos desafios a ser ainda enfrentado pelos pesquisadores da área.

Em 2009 poucos livros de História da educação matemática estavam disponíveis. Nessa época já havia publicação razoavelmente numerosa de artigos em anais de eventos e em periódicos de Educação Matemática e de História da Educação, contudo tal literatura é por vezes de difícil compreensão para os estudantes de graduação.

Por se tratar de uma nova abordagem na disciplina de História da Matemática, foram necessárias algumas edições da disciplina de modo a acomodar temas, bibliografia e estratégias de ensino que possibilitassem efetivamente uma discussão e reflexão significativa aos professores que ensinam matemática.

Três temas podem ser destacados quando se observa as experiências da UFRN e da UFJF, o processo de escolarização da Matemática no 
Brasil e os dois movimentos de renovação do ensino de Matemática que circularam internacionalmente e tiveram suas configurações próprias em nosso país. Esses temas foram já objetos de pesquisas com produção de textos que sistematizam de forma didática os resultados das mesmas, tornando a literatura acessível aos futuros professores.

Passados oito anos dessa reorientação na UFJF, pode-se planejar uma reformulação tanto de ementa, quanto de bibliografia, de modo a incluir outros temas e produções que surgiram posteriormente a partir do próprio desenvolvimento do campo da HEM.

Em 2016, foi aprovada a criação da disciplina História da educação matemática na Universidade Federal de Santa Catarina..$^{41}$ Os temas propostos incluem aqueles tratados na UFJF acrescidos do estudo histórico do curso de licenciatura e da emergência do campo da Educação Matemática. A bibliografia inclui, entre outras obras, livros de História da educação matemática de natureza mais abrangente:

- Schubring, Gert, \& Alexander Karp. Handbook on the History of Mathematics Education. New York: Springer, 2014.

- Valente, Wagner Rodrigues. História da educação matemática no Brasil: problemáticas de pesquisa, fontes, referencias teórico-metodológicas e história elaboradas. São Paulo: Livraria Editora da Física, 2014.

- Garnica, Antonio Vicente Marafioti, \& Luzia Aparecida de Souza. Elementos de história da educação matemática. São Paulo: Editora Cultura Acadêmica, 2012.

Também em 2016, foi aprovada a criação da disciplina de História da educação matemática ${ }^{42}$ na Universidade Federal do Rio Grande do Sul (UFRGS). Os temas abordados incluem a trajetória da Matemática Escolar, aos moldes daqueles apresentados na UFJF, a profissionalização dos professores de Matemática no Brasil e alguma caracterização da pesquisa em História da educação matemática.

\footnotetext{
${ }^{41}$ Disciplina optativa com 4 créditos.

42 Disciplina com 2 créditos.
} 
Nos dois casos anteriormente mencionados, a disciplina de Hem já foi ministrada e a análise dessas experiências iniciais encontra-se publicada em Mendes et al. ${ }^{43}$ Cabe destacar a importância do Repositório de Conteúdo Digital,44 alocado no site da Universidade Federal de Santa Catarina, onde documentos de natureza diversa, incluindo livros didáticos, manuais para professores, provas de alunos, cadernos de alunos e professores, leis e planos de ensino de diferentes épocas e estados brasileiros podem ser acessados. Esses materiais têm sido utilizados nas disciplinas de Hem.

Utilizando a história oral como aporte teórico-metodológico, Silva45 apresenta as iniciativas com a História da educação matemática realizadas na licenciatura em Matemática e no Programa de Pós-Graduação em Educação Matemática da Universidade Estadual de São Paulo (UNESP - Rio Claro). Em nível de graduação, foi criada a disciplina História e Sociologia da Educação: questões da Educação Matemática, e são desenvolvidas intervenções baseadas na história oral que ocorrem em diferentes disciplinas e programas, por exemplo, o Programa Institucional de Bolsas de Iniciação à Docência (PIBID). No Programa de Pós-graduação em Educação Matemática foram inseridas duas disciplinas, História da Educação Matemática Brasileira e História e Educação Matemática: aspectos metodológicos.

Outras experiências de atividades a partir da História da educação matemática não atreladas a uma disciplina e envolvendo alunos da educação básica e de cursos de formação de professores que ensinam matemática foram apresentadas em Souza; Rios \& Silva. ${ }^{46}$ Os autores concluem que

\footnotetext{
${ }_{43}$ Iran Abreu Mendes et al., «A Disciplina História da educação Matemática na Formação de Professores: experiências praticadas ou em andamento», em História da Educação Matemática e Formação de professores, orgs. Bruno Alves Dassiê \& David Antônio da Costa (São Paulo: LF editorial, vol. 1, 2018), 85-120.

${ }_{44}$ Ambiente virtual que reúne documentos digitalizados, que foram garimpados e são mobilizados pelos pesquisadores do GHEMAT. A coordenação do ambiente, que está alocado na UFSC, é do professor David Costa. O acesso ao mesmo é público e gratuito.

${ }_{45}$ Heloisa da Silva, «A História Oral como abordagem em espaços formativos formais de professores de Matemática», Revista de História da Educação Matemática 2 (2016): 162-184.

${ }^{46}$ Luzia Aparecida de Souza, Diogo Franco Rios \& Heloisa da Silva, «O que Pode a História da Educação Matemática em Espaços Não Disciplinares?», em História da Educação Matemática e Formação de professores, orgs. Bruno Alves Dassiê \& David Antônio da Costa (São Paulo: LF editorial, vol. 1, 2018), 121-150.
} 
«O trabalho com diferentes fontes como acervos documentais, conversas e entrevistas criam possibilidades interessantes de estranhamentos sobre escola, educação, matemática, formação, formação de professores... em diferentes comunidades, regimes de ação, tempos».47

Ainda é possível mencionar que pesquisadores do GHEMAT vêm realizando esforços em suas respectivas instituições para a criação da disciplina tanto nos cursos de licenciatura em Matemática como na Pedagogia - é o caso, por exemplo, da Universidade do Estado do Rio de Janeiro (UERJ) e da Universidade Tecnológica Federal do Paraná (UTFPR).

\section{À GUISA DE CONCLUSÃO}

Atualmente no Brasil muitas instituições públicas vêm institucionalizando a História da educação matemática como disciplina no curso de licenciatura em Matemática. Seja como disciplina obrigatória ou eletiva, é caso das universidades federais de Santa Catarina, Rio Grande do Sul, Espírito Santo, Tecnológica do Paraná, Rio Grande do Norte (na qual a disciplina existe desde 2001). Também já ocorre em universidades públicas estaduais tais como Rio de Janeiro e São Paulo.

É importante destacar que os pesquisadores da área provavelmente imprimem em seus cursos, ministrando diferentes disciplinas, marcas, contornos, formas de conduzir a atividade docente determinadas pela atuação na História da educação matemática. Assim, é possível dizer que muitas disciplinas ministradas por esses pesquisadores tem uma abordagem via História da educação matemática.

Contudo, é preciso ponderar sobre a importância da produção de textos, livros de História da educação matemática, que possam ser utilizados como bibliografia em cursos de graduação e pós-graduação de modo a possibilitar que mesmo na falta de especialista na área a disciplina ou os temas possam continuar a serem ministrados.

O campo da Educação Matemática no Brasil vive uma instabilidade, ficando sujeito a contextos locais onde os espaços estão mais garantidos em função das pessoas que os ocupam e a forma como negociam neles

\footnotetext{
${ }_{47}$ De Souza, Diogo Rios \& da Silva, «O que Pode a História da Educação Matemática em Espaços Não Disciplinares?», 148.
} 
a continuidade do que como campo científico com características próprias que define a manutenção de tais espaços. Assim, disciplinas que tragam a marca da Educação Matemática (educação matemática) devem auxiliar na consolidação do campo dentro do ambiente acadêmico na sua relação com o ensino e não só com a pesquisa.

\section{Nota sobre a autora}

Maria Cristina Araújo de Oliveira possui bacharelado e licenciatura em Matemática pela Pontifícia Universidade Católica de São Paulo (1985), mestrado em Matemática pela Universidade de São Paulo (1994), doutorado em Educação (Currículo) pela Pontifícia Universidade Católica de São Paulo (2004) e pós doutorado pela Université de Paris Sud XI (2015). Professora associada do Departamento de Matemática da Universidade Federal de Juiz de Fora, membro do corpo permanente do Programa de Pós-Graduação em Educação Matemática dessa mesma instituição. Atualmente é coordenadora do PPG em Educação Matemática, gestão (2019-2021). É editora da Revista de investigação e divulgação em Educação Matemática (RIDEMA). Participa como pesquisadora do GHEMAT - Grupo de Pesquisa de História da Educação Matemática (www.unifesp.br/centros/ghemat) e do NIDEEM - Núcleo de Investigação, Divulgação e Estudos em Educação Matemática. Tem experiência na área de Educação Matemática, na pesquisa atua principalmente nos seguintes temas: história da educação matemática, formação de professores que ensinam matemática, ensino de geometria. Realizou missão de trabalho na Universidade Nova de Lisboa, em 2006, no âmbito de projeto de cooperação internacional CAPES - GRICES (Brasil-Portugal) (2006-2009). Organizou, em 2010 na UFJF, o IX Seminário Temático A Matemática Moderna nas escolas do Brasil e de Portugal: estudos históricos comparativos.

\section{REFERÊNCIAS}

Boutier, Jean, Jean-Claude Passeron, \& Jacques Revel. Qu'est-ce qu'une discipline?. Paris: École des Hautes Études en Sciences Sociales, 2006.

Fabiani, Jean-Louis. «À quoi sert la notion de discipline?». Em Qu'est-ce qu'une discipline?, editado por Jean Boutier, Jean-Claude Passeron \& Jacques Revel, 11-34. Paris: École des Hautes Études en Sciences Sociales, 2006. 
Goodson, Ivor. «Tornando-se uma matéria acadêmcia: padrões de explicação e evolução». Teoria e Educação 2 (1990): 230-253.

Hofstetter, Rita, \& Bernard Schneuwly. «Disciplinarisation et disciplination consubstantiellement liées. Deux exemples prototypiques sous la loupe: les sciences de l'éducation et des didactiques des disciplines». Em Disziplin-Discipline, editado por Balz Engler, 27-46. Fribourg: Academic Press, 2014.

Hofstetter, Rita, \& Bernard Schneuwly. «Saberes: um tema central para as profissões do ensino e da formação». Em Saberes em (trans)formação: tema central da formação de professores, editado por Rita Hofstetter \& Wagner Rodrigues Valente, 113-172. São Paulo: Livraria da Física, 2017.

Leme da Silva, Maria Célia. «A geometria escolar e o Movimento da Matemática Moderna: em busca de uma nova representação». Em A Matemática Moderna nas escolas do Brasil e de Portugal: contribuição para a história da educação matemática, editado por Cláudia Flores \& Joseane Pinto de Arruda, 6588. São Paulo: Annablume, vol. 1, 2010.

Leme da Silva, Maria Célia, \& Wagner Rodrigues Valente. «Uma breve história do ensinar e aprender matemática nos anos iniciais: uma contribuição para a formação de professores». Educação Matemática Pesquisa 15 (2013): 837855.

Lopes, Marcelo dos Reis. «Matrizes: história de um conteúdo escolar». Dissertação de Mestrado em Programa de Pós-Graduação em Ensino de Matemática, Universidade Federal do Rio de Janeiro, 2012.

Mendes, Iran Abreu. «História da educação matemática na formação de professores de Matemática: uma experiência disciplinar na UFRN». Revista de História da Educação Matemática 2 (2016): 185-199.

Mendes, Iran Abreu, et al. "A Disciplina História da educação Matemática na Formação de Professores: experiências praticadas ou em andamento». Em História da Educação Matemática e Formação de professores, editado por Bruno Alves Dassiê \& David Antônio da Costa, 85-120. São Paulo: LF editorial, 2018.

Oliveira, Alexandre Souza de. «A abordagem do conceito de função em livros didáticos ginasiais: uma análise em tempos modernos (décadas de 1960 a 1970)». Dissertação de Mestrado em Educação Matemática, Universidade Bandeirante de São Paulo, 2009.

Oliveira, Marcus Aldenisson de. «Mudanças nos saberes elementares matemáticos dos anos iniciais: circulação e apropriação do método intuitivo para o ensino de Aritmética (1879-1960)». Tese de Doutorado em Programa de Pós-Graduação em Educação e Saúde, Universidade Federal de São Paulo, 2017.

Oliveira, Maria Cristina Araújo de. «História da educação matemática contributos para a formação de professores que ensinam Matemática». Em Actas IV Congresso Iberoamericano de História da Educação Matemática, editado 
por Dolores Carrillo Gallego et al., 52-59. Murcia: Universidade de Murcia, 2018.

Oliveira, Maria Cristina Araújo de. «Percurso investigativo sobre a geometria e o desenho como saberes profissionais de professores dos anos iniciais no Brasil». Paradigma 39 (2018): 175-189.

Oliveira, Maria Cristina Araújo de. «A. História da educação matemática como disciplina na formação de professores que ensinam Matemática». Cadernos de História da Educação 16 (2017): 653-665.

Oliveira, Maria Cristina Araújo de. "A geometria como disciplina do curso de formação de professores primários: a influência do método intuitivo nas primeiras décadas do século XX no Brasil». Perspectiva 34 (2016): 102-118.

Oliveira, Maria Cristina Araújo de. "O que pesquisas históricas sobre disciplinas de cursos de licenciatura em Matemática brasileiros revelam sobre essa formação?». Jornal Internacional de Estudos em Educação Matemática 6 (2013): 144-165.

Pinheiro, Nara Vilma Lima. «A aritmética sob medida: a matemática em tempos da pedagogia científica». Tese de Doutorado em Programa de Pós-Graduação em Educação e Saúde, Universidade Federal de São Paulo, 2017.

Silva, Heloisa da. "A História Oral como abordagem em espaços formativos formais de professores de Matemática». Revista de História da Educação Matemática 2 (2016): 168-184.

Souza, Luzia Aparecida de, Diogo Franco Rios \& Heloisa da Silva. «O que Pode a História da Educação Matemática em Espaços Não Disciplinares?». Em História da Educação Matemática e Formação de professores, editado por Bruno Alves Dassiê \& David Antônio da Costa, 121-150. São Paulo: LF editorial, v. 1, 2018.

Valente, Wagner Rodrigues. «Saber objetivado e formação de professores: reflexões pedagógico-epistemológicas». História da Educação 23 (2019): 1-22.

Valente, Wagner Rodrigues. «História da educação matemática: considerações sobre suas potencialidades na formação do professor de matemática». Bolema 23 (2010): 123-136.

Valente, Wagner Rodrigues. «O Lugar da Matemática Escolar na Licenciatura em Matemática». Bolema 27 (2013): 939-955.

Valente, Wagner Rodrigues. "O saber profissional do professor que ensina matemática: história da matemática a ensinar e da matemática para ensinar em construção». Em História da Educação Matemática e Formação de professores, editado por Bruno Alves Dassiê \& David Antônio da Costa, 49-84. São Paulo: LF editorial, v. 1, 2018.

Valente, Wagner Rodrigues. Euclides Roxo e a modernização do ensino de Matemática no Brasil. São Paulo: Editora da Sociedade Brasileira de Educação Matemática, 2003. 
Valentim Júnior, Josélio Lopes. «A geometria analítica como conteúdo do ensino secundário: análise dos livros didáticos utilizados entre a Reforma Capanema e o MMM». Dissertação de Mestrado em Educação Matemática, Universidade Federal de Juiz de Fora, 2013.

Vidal, Diana Gonçalves. Grupos escolares: cultura escolar primária e escolarização da infância no Brasil (1893-1971). Campinas, SP: Mercado das Letras, 2006. 